\title{
Underwater endoscopic mucosal resection of a condyloma acuminatum of the anal canal
}

\author{
Kenta Hamada ${ }^{a}$, Noriya Uedo ${ }^{a}$, Yasuhiko Tomita ${ }^{b}$, Ryu Ishihara ${ }^{a}$ \\ Osaka Medical Center for Cancer and Cardiovascular Diseases, Osaka, Japan
}

A man in his 70s presented to our hospital with a positive fecal occult blood test. Colonoscopy revealed a $12-\mathrm{mm}$ protruding lesion in the anal canal (Fig. 1 A,B). Because the anal canal was too narrow to perform conventional endoscopic mucosal resection (EMR), underwater EMR without submucosal injection was performed using a colonoscope (CF-HQ290I; Olympus, Tokyo, Japan). A disposable transparent attachment (D-201-14304; Olympus) was mounted on the tip of the colonoscope. The rectal lumen was completely deflated and the lesion in the anal canal was completely immersed in water by use of a mechanical pump (OFP; Olympus). A polypectomy snare $(15 \mathrm{~mm}$, Captivator II, Boston Scientific, Marlborough, MA, USA) was used. The lesion was removed en bloc with electric current (VIO300D, Erbe Elektromedizin $\mathrm{GmbH}$, Tübingen, Germany Endocut mode, Effect 3, Duration 3, Interval 3) (Fig. 1C). The resection wound was closed with hemoclips. The patient experienced no adverse events. Histological examination showed condyloma acuminatum (Fig. 1D).

Condyloma acuminatum of the anal canal is a very common sexually transmitted disease caused by human papilloma virus infection. Although generally regarded as benign, condyloma acuminatum is difficult to treat, and recurrence is frequent following surgical removal [1,2]. Successful endoscopic submucosal dissection has been reported [2], but in the present case the anal canal was too narrow, making endoscopic submucosal dissection technically difficult. Moreover,

Departments of ${ }^{a}$ Gastrointestinal Oncology (Kenta Hamada, Noriya

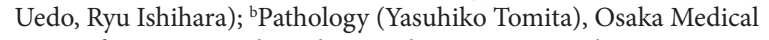
Center for Cancer and Cardiovascular Diseases, Osaka, Japan

Conflict of Interest: None

Correspondence to: Noriya Uedo, Department of Gastrointestinal Oncology, Osaka Medical Center for Cancer and Cardiovascular Diseases, 1-3-3 Nakamichi Higashinari-ku, Osaka 537-8511, Japan, Tel.: +81 66972 1181, Fax: +81 66981 4067,

e-mail: uedou-no@mc.pref.osaka.jp

Received 10 August 2016; accepted 14 August 2016; published online 7 October 2016

DOI: https://doi.org/10.20524/aog.2016.0095

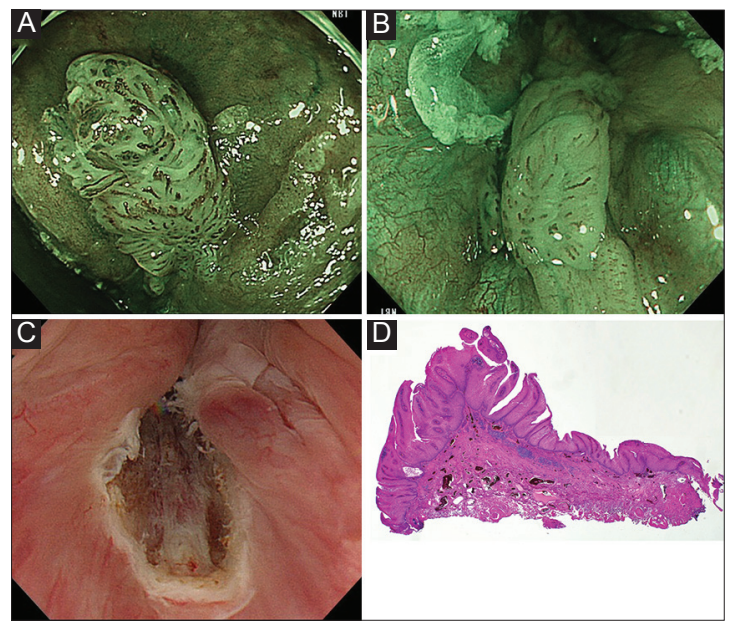

Figure $1(\mathrm{~A}, \mathrm{~B})$ Colonoscopy showed a $12-\mathrm{mm}$ protruding lesion in the anal canal. (A) The rectal side of the lesion. (B) The anal side of the lesion. (C) The wound just after underwater endoscopic mucosal resection of the lesion in the anal canal. (D) Histological examination showed condyloma acuminatum

submucosal injection may interfere with the limited working space and make the EMR procedure difficult. Underwater EMR obviates the need for submucosal injection [3] and facilitates the endoscopic resection of a lesion in the anal canal. Additionally, water immersion provides a natural magnification effect and enhances the topography of the mucosa, facilitating identification of the remnant just after resection.

\section{References}

1. Blomberg M, Friis S, Munk C, Bautz A, Kjaer SK. Genital warts and risk of cancer: a Danish study of nearly 50000 patients with genital warts. J Infect Dis 2012;205:1544-1553.

2. Sasaki A, Nakajima T, Egashira H, et al. Condyloma acuminatum of the anal canal, treated with endoscopic submucosal dissection. World J Gastroenterol 2016;22:2636-2641.

3. Binmoeller KF, Weilert F, Shah J, Bhat Y, Kane S. "Underwater" EMR without submucosal injection for large sessile colorectal polyps (with video). Gastrointest Endosc 2012;75:1086-1091. 